\title{
Dynamic foot morphology explained through 4D scanning and shape modeling
}

\author{
Abhishektha Boppana ${ }^{\mathrm{a}, *}$, Allison P. Anderson ${ }^{\mathrm{a}}$ \\ ${ }^{a}$ Ann and H.J. Smead Department of Aerospace Engineering Sciences, University of \\ Colorado Boulder, USA
}

\begin{abstract}
A detailed understanding of foot morphology can enable the design of more comfortable and better fitting footwear. However, foot morphology varies widely within the population, and changes dynamically during the loading of stance phase. This study presents a parametric statistical shape model from 4D foot scans to capture both the inter- and intra-individual variability in foot morphology. Thirty subjects walked on a treadmill while 4D scans of their right foot were taken at 90 frames-per-second during stance phase. Each subject's height, weight, foot length, foot width, arch length, and sex were also recorded. The $4 \mathrm{D}$ scans were all registered to a common high-quality foot scan, and a principal component analysis was done on all processed 4D scans. Elastic-net linear regression models were built to predict the principal component scores, which were then inverse transformed into $4 \mathrm{D}$ scans. The best performing model was selected with leave-one-out cross-validation. The chosen model was predicts foot morphology across stance phase with a root-mean squared error of $5.2 \pm 2.0$ $\mathrm{mm}$. This study shows that statistical shape modeling can be used to predict dynamic changes in foot morphology across the population. The model can be used to investigate and improve foot-footwear interaction, allowing for better fitting and more comfortable footwear.
\end{abstract}

Keywords: foot morphology, dynamic scanning, gait biomechanics, shape modeling

\section{Introduction}

Foot shape is known to be highly variable throughout the population, including by sex (Wunderlich and Cavanagh, 2001; Krauss et al., 2008, 2010), age

\footnotetext{
* Corresponding Author

Email address: abhishektha@colorado.edu (Abhishektha Boppana)
} 
(Tomassoni et al., 2014), and weight (Price and Nester, 2016). This variability is often not captured in footwear sizing, as current footwear fitting standards only use foot length, foot width, and arch length to fit to standardized shoe sizes (ASTM F539-01, 2017). Furthermore, footwear is commonly designed around lasts, shoe molds that are sized and shaped by each manufacturer with no common standard, leading to variability in footwear shapes and sizes (Jurca and Dzeroski, 2013; Wannop et al., 2019). Such variability can make it hard for consumers to find a proper fit, resulting in users having to wear ill-fitting footwear with suboptimal comfort (Dobson et al. 2018). Footwear comfort has shown benefits in increasing running performance (Luo et al., 2009) and reducing the risk of movement-related injury (Mündermann et al. |2001), and is often the number one (Martínez-Martínez et al., 2017) factor for consumers to select footwear. Footwear should therefore be properly fit to a wide population range in order to be successful.

However, because the current methodology of designing footwear relies on using static lasts, this assumes that the foot consists of rigid segments. This fails to account for dynamic changes in foot morphology, especially when the foot is being loaded during gait. Assumptions of rigid foot segments during foot loading have shown inaccuracies in estimation of ankle joint mechanics (Zelik and Honert, 2018; Kessler et al., 2020), suggesting intra-foot motion as the foot is loaded (Lundgren et al., 2008; Wolf et al., 2008). Evidence suggests that foot loading affects linear foot measurements, such as when transitioning from sitting to standing (Xiong et al., 2009, Oladipo et al., 2008) or during the stance phase of gait (Kouchi et al. | 2009; Barisch-Fritz et al., 2014a, Grau and Barisch-Fritz, 2018). The dynamically changing measurements suggest morphological changes occurring, all of which may not be captured in static linear and circumferential measurements. Thus, it becomes difficult to characterize the wide variety of foot shapes across not only a large population, but within individuals as their foot goes through loading scenarios such as gait.

Statistical shape models (SSMs) can explain morphological differences across populations by identifying shape modes which account for variance from the mean foot,. These have been developed for whole-body digital human modeling applications to study population and individual variance in body shape (Allen et al., 2003, Anguelov et al., 2005, Reed et al., 2014, Park and Reed, 2015, Park et al. 2017). Parametric SSMs are extensions which use correlations between subject anthropometric data and SSM deformations to help predict body shape for new individuals in the population (Park and Reed, 2015; Park et al., 2017).

SSMs have recently been applied to characterize static foot shape across a population (Conrad et al., 2019) and recognize foot-shape deviations (Stanković et al. 2020). The aforementioned efforts to capture foot measurement changes over the gait cycle did capture 4D foot images (Barisch-Fritz et al. 2014a Grau and Barisch-Fritz, 2018), but these efforts were not translated into a SSM. All the previously developed systems were also based on a catwalk, requiring subjects to correctly hit the scanning area for a successful data capture, which may not be representative of natural cadence.

The development of the DynaMo software (Boppana and Anderson, 2019) 
for the Intel RealSense D415 Depth Cameras (Intel, Santa Clara CA) allowed a $4 \mathrm{D}$ scanning system to be set around a treadmill, where subjects can maintain a natural cadence. This system captures the majority of the foot's dorsal surface, but does not allow for the capture of the foot's plantar surface. 4D scans are captured at $90 \mathrm{fps}$, enabling a detailed evaluation of foot morphology changes during loading and unloading. This study outlines the development of a parametric SSM, derived from scans captured with this system. The parametric SSM can characterize and predict dynamic foot morphology at specific points during stance phase across the subject population. We hypothesize that there will be significant changes in foot morphology across the dorsal surface of the foot throughout the gait cycle. We also hypothesize that these changes will be predictable from the subject demographics of our population.

\section{Methods}

\subsection{Subjects}

A total of 30 healthy subjects (15 men and 15 women, ages $23.1 \pm 3.7$ ) participated in this study. Subjects were recruited in a stratified sample into one of six groups ( 5 subjects per group) to maximize variance in population foot length. Height was used as the grouping factor since height is well correlated to foot length (Giles and Vallandigham, 1991). The general population may not know offhand their exact foot length, and shoe size varies by manufacturer and does not correspond directly to foot length (Jurca and Dzeroski, 2013. Wannop et al., 2019). Groups consisted of 5th-35th, 35th-65th, and 65th-95th height percentiles for each sex. Height percentile values were taken from the ANSUR II survey (Gordon et al., 2014) and converted to imperial units as it was expected most subjects would report their height in imperial units. Population recruitment groups are summarized in tbl. 1.

Prior to recruitment, subjects completed a prescreening survey to ensure they were adequately healthy by the American College of Sports Medicine guidelines(Riebe et al. 2015), and between the ages of 18-65. Subjects provided their sex and height, and were only enrolled in the study if their population group was not fully enrolled.

\subsection{Experimental Procedures}

The experimental protocol was approved by the University of Colorado Institutional Review Board. Procedures were explained to each subject and written consent was obtained prior to participation. Subjects' height and weight were recorded with a tape measure and scale, respectively. Subjects' foot length, foot width, and arch length were measured with a Brannock device (The Brannock Device Company, Liverpool, NY) (ASTM F539-01, 2017). Both foot length and arch length were measured in centimeters. Foot width was measured as an ordinal size (e.g. A, B, C, D, E), and then converted to a linear measurement in centimeters (The Brannock Device Company, Liverpool, NY). 
Six Intel RealSense D415 Depth Cameras (Intel, Santa Clara, CA) were placed and calibrated around a custom-built level treadmill in the University of Colorado Boulder Locomotion Laboratory, as shown in Fig. 1. The DynaMo software package was used to capture depth images of the right foot at 90 frames-per-second while subjects walked on the treadmill, and convert each frame's depth images to a single point cloud (Boppana and Anderson, 2019).

The treadmill was set to an average walking pace of $1.4 \mathrm{~m} / \mathrm{s}$ (Browning et al. 2006). Reflective markers were placed on the subject's right foot and a black sock over their left foot to aid in right foot identification. Subjects first walked for one minute to warm-up and fall into a natural cadence. The operator then collected 10 seconds of data to capture approximately 10 steps. The data were reviewed to ensure the subject stayed in frame from heel-strike to toe-off during capture. If needed, the subject's placement was shifted and data was collected again, up to two times.

\subsection{Data Processing}

(Fig. 2 provides an overview of the data processing workflow. The following paragraphs summarize the workflow, while more detail is provided in supplementary methods.

For each subject, a candidate heel-strike to toe-off event was manually identified across all captures by taking into account point cloud quality due to the high computational power required to process all heel-strike to toe-off events. The depth images captured by each depth camera were processed into point clouds using the DynaMo package (Boppana and Anderson, 2019). From each point cloud, the right foot was isolated and transformed into a triangle mesh (Rusu and Cousins, 2011; Fischler and Bolles, 1981; Bernardini et al., 1999 Zhou et al. | 2018). Since every depth image was captured independently by the cameras, the amount and location of points which represented the foot were not consistent. In addition, the captured data may have holes in the surface representing the foot. Registration of all scans to a common template represents every scan by an equal number of points, and ensures any missing points are properly interpolated. The right foot meshes were then iteratively registered using a three-step fitting process to an averaged high-quality static template scan from a previous study (Reed et al., 2013). First scans were roughly aligned using a point-to-plane iterative-closest-point algorithm (Chen and Medioni, 1992), implemented in Open3D (Zhou et al., 2018). Next, the radial-basis function fitting algorithm from the GIAS2 software package (Zhang et al., 2016) was run twice using a thin-plate spline to approximate the foot surface (Park and Reed, 2015 Kim et al., 2016). The mid-stance scan from each subject was registered first to the template, and then the registration process was run both forwards towards toe-off and backwards towards heel-strike, on a scan-by-scan basis, using the previously registered scan as a template for the next scan. Accuracy was checked by comparing registered scans with the processed scans by finding corresponding points between both, and calculating the root-mean-squared error (RMSE) between the corresponding points. 
Anatomical landmarks can be reliably approximated from the registered scans (Van den Herrewegen et al., 2014). The first metatarsal head, fifth metatarsal head, and second toe landmarks were used to align all scans to be centered at the second metatarsal head, with the forward axis pointing towards the second toe. Landmarks around the metatarsal-phalangeal (MTP) joint and ankle joint were used to calculate ankle, MTP, and foot kinematics for each subject's scans with respect to the joint angles at the subject's mid-stance scan. Relevant joint angles include dorsi/plantarflexion, ankle inversion/eversion, ankle internal/external rotation, MTP dorsi/plantarflexion, foot inversion/eversion, and foot internal/external rotation angles

\subsection{Model Construction}

Principal component (PC) analysis is a dimensionality-reduction method commonly in constructing SSMs (Reed and Parkinson, 2008; Park and Reed, 2015, Conrad et al. 2019, Stanković et al., 2020). The first PC represents an axis containing the largest variance in the dataset, and each subsequent $\mathrm{PC}$ describes the largest variance orthogonal to the previous component's axis. Therefore, PCs allow for a new, smaller set of orthogonal variables to be defined which represent the variance in the dataset.

Let $N$ equal the number of total scans in the dataset, and $n=29873$ equal the number of vertices in each registered scan. The scikit-learn module (Pedregosa et al. (2011) was used to incrementally calculate the maximum $N$ PCs which represent the dataset. Each scan in the dataset is represented in the PC model with $N$ PC scores. All PC scores are centered around 0, which represents the mean foot scan of the dataset containing all subjects. Each PC represents a shape mode in the SSM, where each score represents a deviation from the mean foot along the shape mode axis. The resultant PC model can be used to inverse transform a vector of length $N$ PC scores into a $29873 \times 3$ vector, which represents the location of the vertices in the foot shape. Not all PCs were retained in the model since the first few PCs explain a majority of the variance, while additional PCs may be accounting for noise.

Subject demographic data and calculated joint angles were incorporated into the SSM by developing multivariate linear regression models based on these features. This was used to predict each PC score, which can then be inversetransformed into a foot shape. Subject demographic data and joint angles were normalized and power-transformed to aid in regression development (Yeo and Johnson, 2000). An elastic net regularization algorithm (Zou and Hastie, 2005) was run for each multivariate regression to calculate normalized feature coefficients for each PC score's regression. Two different sets of predictors were created, one with all subject demographic data and calculated joint angles, and one with the highly cross-correlated predictors of arch length, body-mass index,

and height were removed (see Supplementary Figures). Six potential models were built as combinations between the number of PCs predicted which explained $95 \%, 98 \%$, and $99.7 \%$ of the variance, and the two predictor sets. 


\subsection{Model Validation}

All six models were validated for performance using leave-one-out crossvalidation, where scans from each subject were set as the validation set, and models were trained on the remaining dataset. Model performance during validation was quantified with the root mean squared error (RMSE) of the predicted foot shape to the corresponding registered scan. A two-way RMANOVA analysis was run on the error distributions to test the effect of constructing a predictor with the different number of PCs, and between using the two variable sets. The chosen model was retrained on the whole dataset before being analyzed. \# Results

A total of 1771 scans were analyzed across all 30 subjects. Each subject's stance phase ranged from 52-69 scans (mean=59). (Fig. 3) shows a set of raw and registered scans from one subject. All processed scans were registered to the template with a median registration accuracy of $1.0 \pm 0.6 \mathrm{~mm}$.

The PCA analysis of all registered scans found the first 8 PCs to represent approximately $95 \%$ of the variance, the first 27 PCs to represent approximately $98 \%$ of the variance, and the first 105 PCs to represent approximately $99.7 \%$ of the variance. (Fig. 4 ) shows the distribution of cross-validation RMSEs for each of the six elastic net regression models tested. RMSE distributions did not meet assumptions for normality, but RMANOVA was still used to compare models due to its resiliency to deviations from normality. A significant difference was found between predicting different numbers of PCs $(\mathrm{F}=1595.0, \mathrm{p}<0.001)$, predicting between the two variable sets $(\mathrm{F}=81.6, \mathrm{p}<0.001)$, and the interaction between both factors $(\mathrm{F}=213.7, \mathrm{p}<0.001)$. Significant differences were found between all three levels of the predicted number of PCs $(p$-adj $<0.001)$ with a Tukey post-hoc HSD test. No significant difference was found between the two variable sets $(\mathrm{p}$-adj $=0.42)$. Therefore, the model predicting $8 \mathrm{PCs}$ with the selected variable set was chosen for its simplicity and performance.

Each retained PC is a shape mode in the model. (Fig. 5) shows the chosen model's normalized regression coefficient values for each shape mode. The coefficients for the sex predictor are not shown as they were calculated to be zero for every shape mode.

(Fig. 6) shows each shape mode's axis represented on the mean foot, highlighting which areas of the foot are affected by deformations in each shape mode. (Fig. 7) shows the \pm 2 standard deviations of deformation along each shape mode overlaid on the mean foot. Supplementary information includes correlation between figures, ratio of total variance each retained PC accounts for, and a video showing the predictive capability of the model.

\section{Discussion}

This study was designed to construct and evaluate a parametric SSM in explaining and predicting dynamic foot morphology changes across the subject population. The model was able to predict dynamic foot shape across the subject population with an average RMSE of $5.2 \pm 2.0 \mathrm{~mm}$. For context, if all possible prediction error was accumulated to only affect length and 
width, it would be higher than the half-size step of the American shoe sizing system (Luximon and Luximon, 2013), but less than inter-brand variability of shoe length and shoe width (Wannop et al., 2019). Further, this error is lower than the RMSEs of other parametric SSMs that predicted static standing child body shape (mean $=10.4 \mathrm{~mm})$ (Park and Reed, 2015), dynamic shoulder deformation $($ mean $=11.98 \mathrm{~mm})$ (Kim et al. 2016$)$ and child torso shape $($ mean $=9.5 \mathrm{~mm})$ (Park et al. 2017). Note though, that the presented model may have lower prediction errors due to the foot being a relatively smaller section of the body to model. Grant et al's model reconstructed internal foot bones with much lower RMSEs from sparse anatomical landmarks (1.21-1.66 $\mathrm{mm}$ for various foot segments) (Grant et al. 2020) but was trained with higher resolution MRI images. Other efforts to create statistical foot shape models did not incorporate parametric prediction of foot shape (Conrad et al., 2019, Stanković et al., 2020).

The first, second, and fourth shape modes, accounting for a total of $86.7 \%$ of total variance, capture gross foot motion. Foot motion during stance is dominated by MTP and ankle dorsi/plantarflexion (Leardini et al., 2007), which is captured in the first shape mode (Fig. 7). The second and fourth shape modes capture gross changes in foot rotation from frontal and transverse plane movements at the MTP and ankle joints, respectively (Fig. 7). The second shape mode is most affected by foot inversion/everison around the MTP joint. The second shape mode also captures girth scaling at the ankle joint, as seen in (Fig. 7) by how the ankle girth decreases along the axis, and is affected by weight (Fig. 5). The fourth shape mode is affected by ankle inversion/eversion and internal/external rotation. Foot inversion/eversion, ankle inversion/eversion, and ankle internal/external rotation are expected to vary across the stance phase ((Leardini et al. 2007)), which leads to the observed changes in gross movement. However, the second and fourth shape modes are slightly affected by foot length, which may suggest inter-individual effects in foot inversion/eversion, ankle inversion/eversion, and internal/external rotation during gait. There is a slight correlation between these angles and foot length (see supplementary figures), which may be due to differences in cadence when walking at the treadmill's set speed. Individuals were given time to acclimate to the treadmill's set speed, but the speed may not have been their preferred walking speed.

The third shape mode captures foot shape scaling at the rearfoot, as highlighted in (Fig. 6). Foot length shrinks when moving positively along the third shape mode (Fig. 7), and thus has a negative effect from foot length. There are also negative effects from foot width and weight, which may be due to their correlation to foot length (see supplementary figures). Rearfoot morphology along this shape mode has a more rounded shape in the negative direction, and a sharper shape in the positive direction (Fig. 7). There is also a negative effect from foot inversion/eversion (Fig. 5), indicating that with foot eversion, a sharper rearfoot shape is expected. This may be due to foot eversion at heel-off (Leardini et al. 2007), where the foot unloads from a rounder weight-bearing rearfoot to a sharper non-weight bearing rearfoot shape.

Midfoot girth increases and the rearfoot is rounder along the fifth shape mode's axis (Fig. 7). The fifth shape mode is positively affected by foot length 
and negatively by MTP dorsi/plantarflexion (Fig. 5). This suggests that static midfoot girth increases with foot length, and decreases through heel-off as the MTP dorsiflexes. Rearfoot morphology is rounder for longer foot lengths but gets sharper through heel-off with MTP dorsiflexion, much like in the third shape mode. Midfoot girth was previously found to decrease during stance phase compared to statically standing (Grau and Barisch-Fritz, 2018), most likely due to intrinsic and extrinsic foot muscle contraction (Scott and Winter, 1993: Gefen et al., 2000). However, it was not noted where during stance phase midfoot girth decreases, but it can now be assumed it occurs during heel-off.

The sixth shape mode captures girth changes at the ankle, midfoot, and the medial MTP joint region (Fig. 6), with girth increasing along the axis. There are positive effects from ankle internal/external rotation and weight, while there is a negative effect from ankle inversion/eversion (Fig. 5). Static MTP, midfoot, and ankle girth may therefore increase with subject weight. Dynamic girth changes in these regions may occur as the ankle everts and internally rotates just prior to toe-off, where muscle activation is needed to push the foot off the ground. The foot is stiffened through tension in the MTP joints in order to prepare for toe-off (Hicks, 1954), and the MTP joints are known to move relatively within the foot during gait (Wolf et al., 2008; Lundgren et al., 2008) which may be resulting in the increased girth at the MTP joint. A similar mechanism may be occuring at the ankle joint during ankle inversion and internal rotation, where tension from muscle activation prior to toe-off may cause increased girth.

The seventh and eight shape modes, accounting for $1.3 \%$ of total variance, capture girth increases near the medial malleolus along their axes (Fig. 6). They are both positively affected by ankle inversion/eversion (Fig. 5), and the eight shape mode is further negatively affected by ankle internal/external rotation. This may suggest that the girth around the medial malleolus decreases prior to push-off, as the ankle everts and internally rotates.

Observed girth changes at the ankle joint, medial malleolus, midfoot, and MTP joint can be directly mapped to footwear design recommendations for increased fit and comfort. Midfoot girth decreased as the MTP joint is dorsiflexing after heel-off. Midfoot, ankle, and MTP joint girth increased and medial malleolus girth decreased through ankle eversion and external rotation just prior to toe-off. Footwear should be designed to follow these volume changes as the footwear itself goes through the same motions, to ensure proper support for the foot to drive the footwear through the stance phase and toe-off. For example, footwear may be designed to first contract as the MTP joint dorsiflexes, then subsequently expand around the midfoot, ankle and MTP joints while contracting around the medial malleolus as the ankle everts and externally rotates.

A number of limitations in this study should be noted. The elastic-net method is able to retain cross-correlated predictors, but still requires some bias in the dataset to predict scenarios where cross-correlated predictors are independent (Zou and Hastie, 2005). Therefore, the presented model may not be valid for predicting changes in morphology due to independent changes in joint angles outside of stance phase, or for variance in foot width or weight compared to foot length not captured in the subject population. 
The model did not capture differences between male and female feet. Studies found that sex differences in foot shape after scaling for foot length were not significant (Kouchi et al. 2009, Barisch-Fritz et al., 2014b: Conrad et al., 2019), or were small in magnitude (Wunderlich and Cavanagh, 2001; Krauss et al., 2008). No subject demographic data was collected to account for differences in foot shape due to ethnicity (Jurca et al. 2019). No data was captured on the foot's plantar surface due to limitations with the scanning system; therefore foot arch changes were not captured. Data captured around the toes had high noise, which necessitated smoothing the toes in the template to ease fitting. Future advances in $4 \mathrm{D}$ scanning may alleviate some of these concerns, and also allow for expansion of this model to higher frequency foot motions, such as running.

\section{Conclusions}

To the authors' knowledge, this is the first parametric foot SSM that captures and reconstructs dynamic motion. The model was able to identity specific changes in foot morphology as they related to subject and kinematic parameters, and suggest footwear design techniques to increase fit and comfort. The model is able to reconstruct a full 3D model when parameter values are provided, which offers shoe and last designers a design starting point, and the ability to test their designs on a range of subjects throughout stance phase. 


\section{Figures and Tables}

All tables, figures, and respective captions are listed below

Table 1: Enrollment groups based on reported height. 5 subjects were enrolled in each group

\begin{tabular}{|c|c|c|c|}
\hline Sex & $\begin{array}{l}\text { 5th-35th percentile } \\
\text { Height }\end{array}$ & $\begin{array}{l}35 \text { th-65th percentile } \\
\text { Height }\end{array}$ & $\begin{array}{l}\text { 65th-95th percentile } \\
\text { Height }\end{array}$ \\
\hline Femal & e 4'11“-5’3" & $5 ’ 3 “-5 ’ 5 "$ & $55^{\prime \prime}-5 ’ 8 "$ \\
\hline Male & $5 ' 4^{\prime \prime}-5 ' 8 "$ & $5 ' 8 “-5 ' 11 "$ & $5 ' 11^{\prime \prime}-6 ’ 2 "$ \\
\hline
\end{tabular}




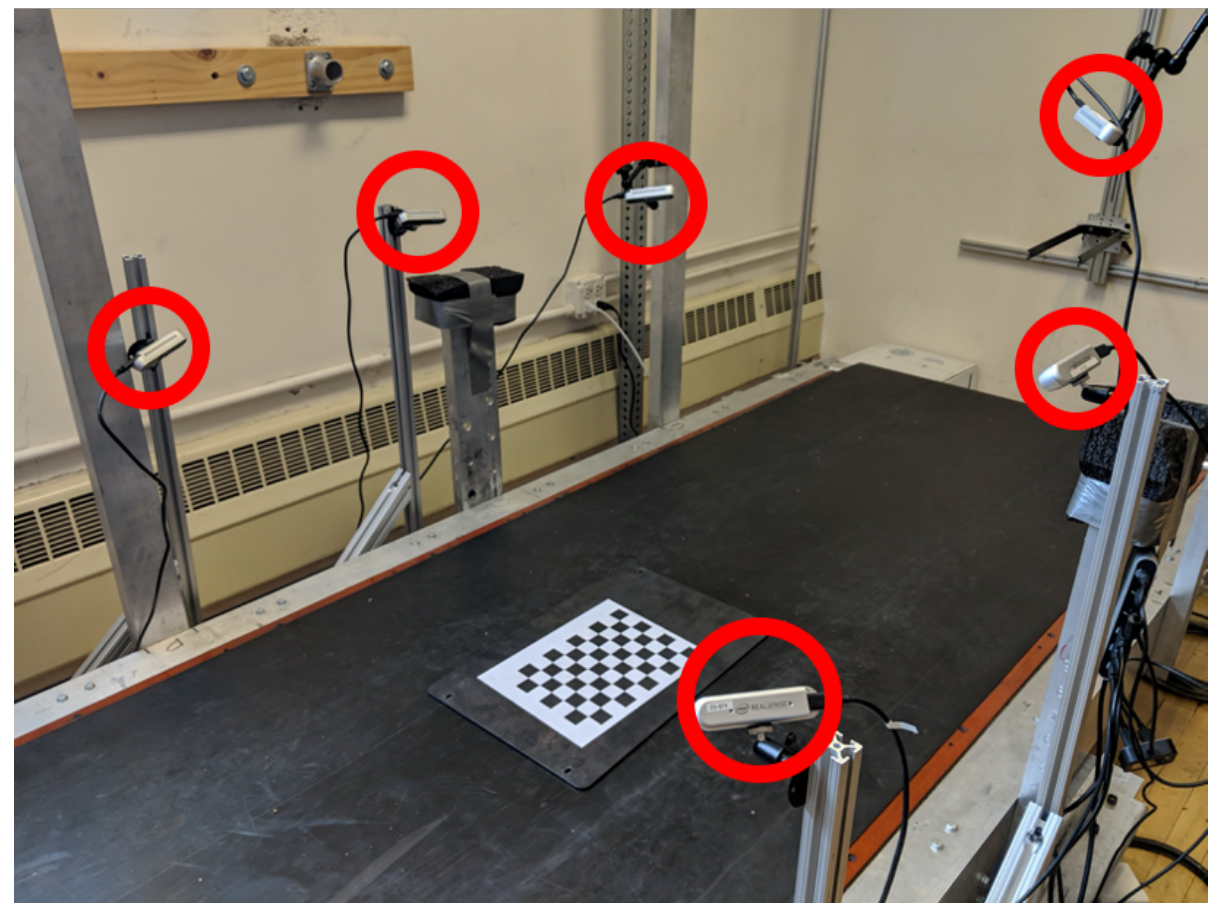

Figure 1: Capture setup of 6 Intel RealSense D415 Depth Cameras (circled in red) placed around a treadmill. The checkerboard shown was used to calibrate the cameras using the DynaMo package. 


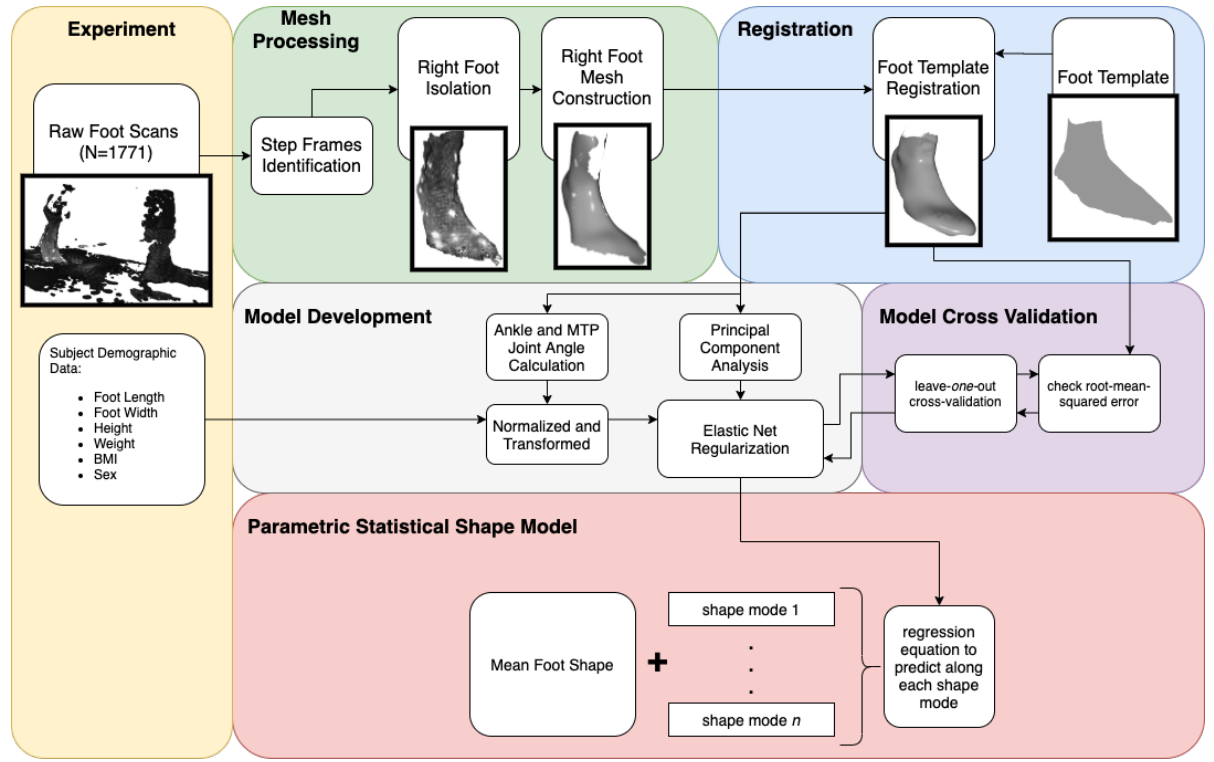

Figure 2: Flowchart of processing steps for statistical shape model creation 


\section{Processed Scans}
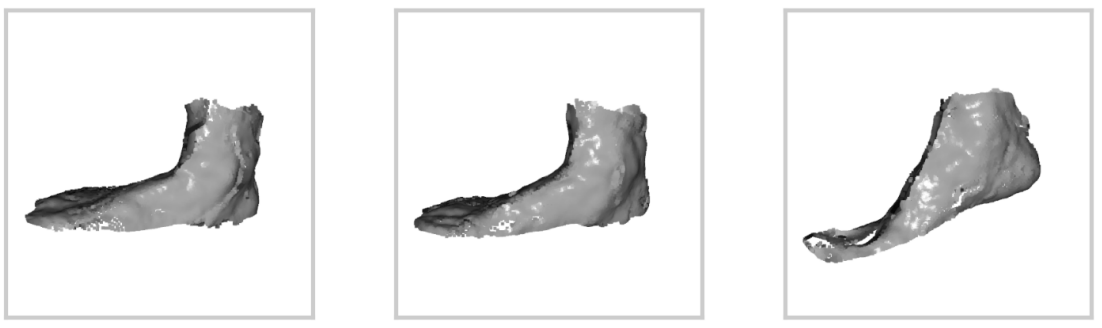

\section{Registered Scans}

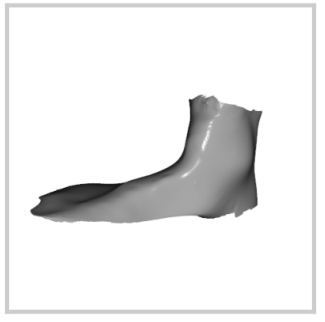

$0 \mathrm{~s}$

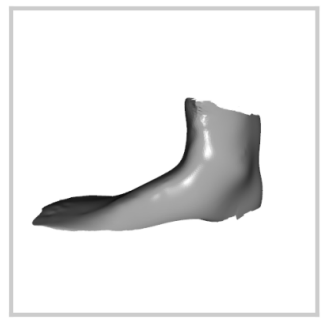

$+.11 \mathrm{~s}$

(after heel-off)

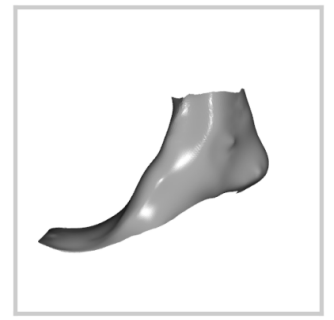

$+.22 \mathrm{~s}$

(before toe-off)

(approximately mid-stance)

Figure 3: Processed and registered scans of one subject during heel-off, shown 10 frames (.11 seconds) apart 


\section{Performance of Prediction Models with Leave-Subject-Out Cross-Validation}

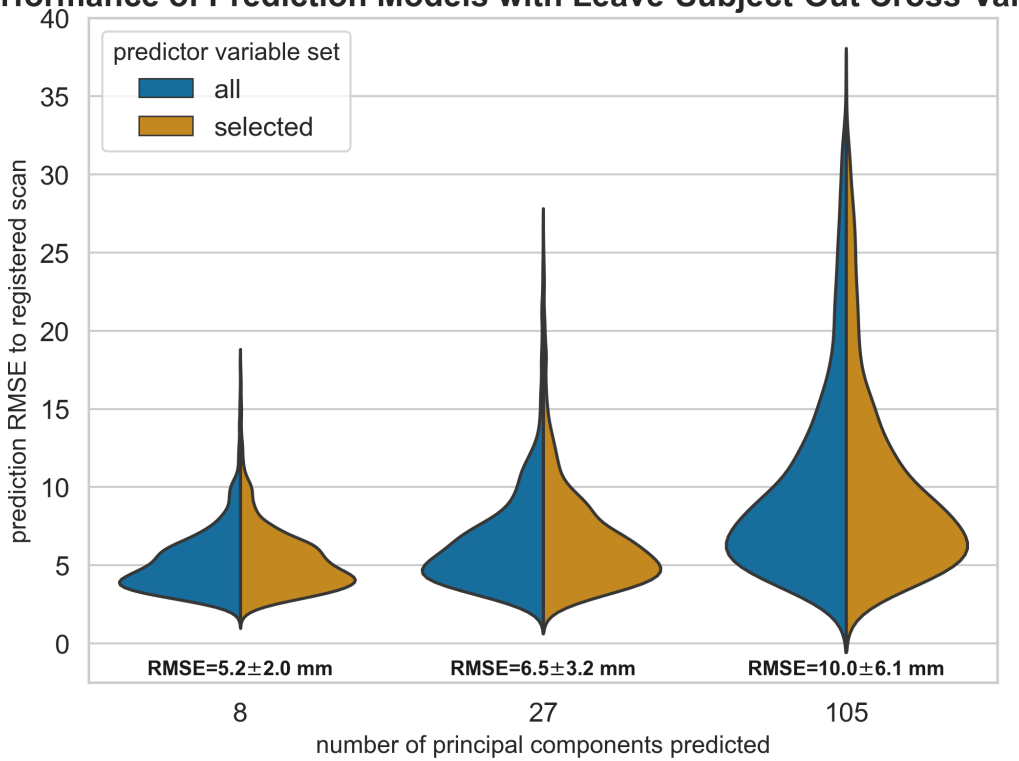

Figure 4: Distribution of errors across the various prediction models leave-subject-out crossvalidation results. Model RMSE mean and standard deviation are shown above each distribution 
Normalized Regression Coefficients per Shape Mode
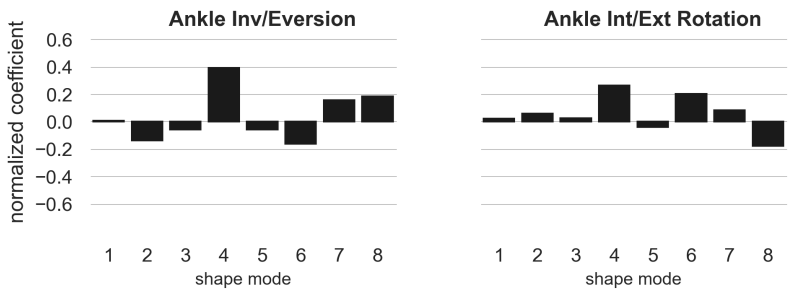

Ankle Dorsi/Plantarflexion

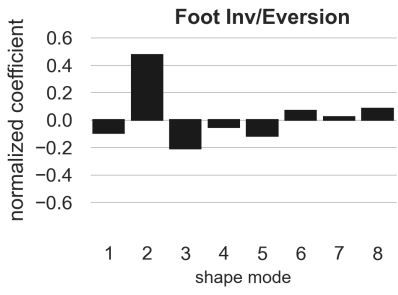

Foot Int/External Rotation

$\begin{array}{llllllll}1 & 2 & 3 & 4 & 5 & 6 & 7 & 8\end{array}$

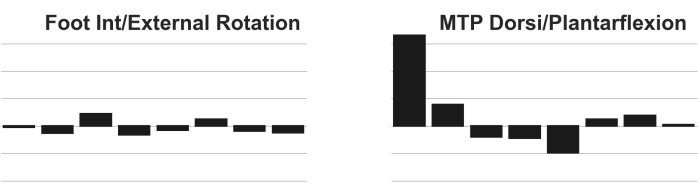

$\begin{array}{lllllll}2 & 3 & 4 & 5 & 6 & 7 & 8\end{array}$

$\begin{array}{llllllll}1 & 2 & 3 & 4 & 5 & 6 & 7 & 8\end{array}$

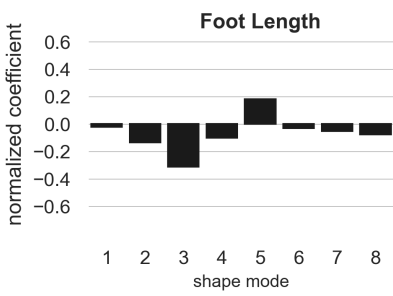

Foot Width

Weight
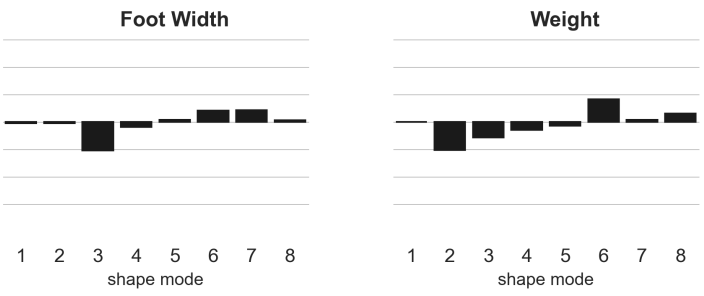

Figure 5: Each graph represents the predictor's effects on the shape mode by visualizing the model's normalized coefficients. Larger absolute values indicate a larger effect from the predictor on the shape mode. 


\section{Areas of foot affected by each shape mode}
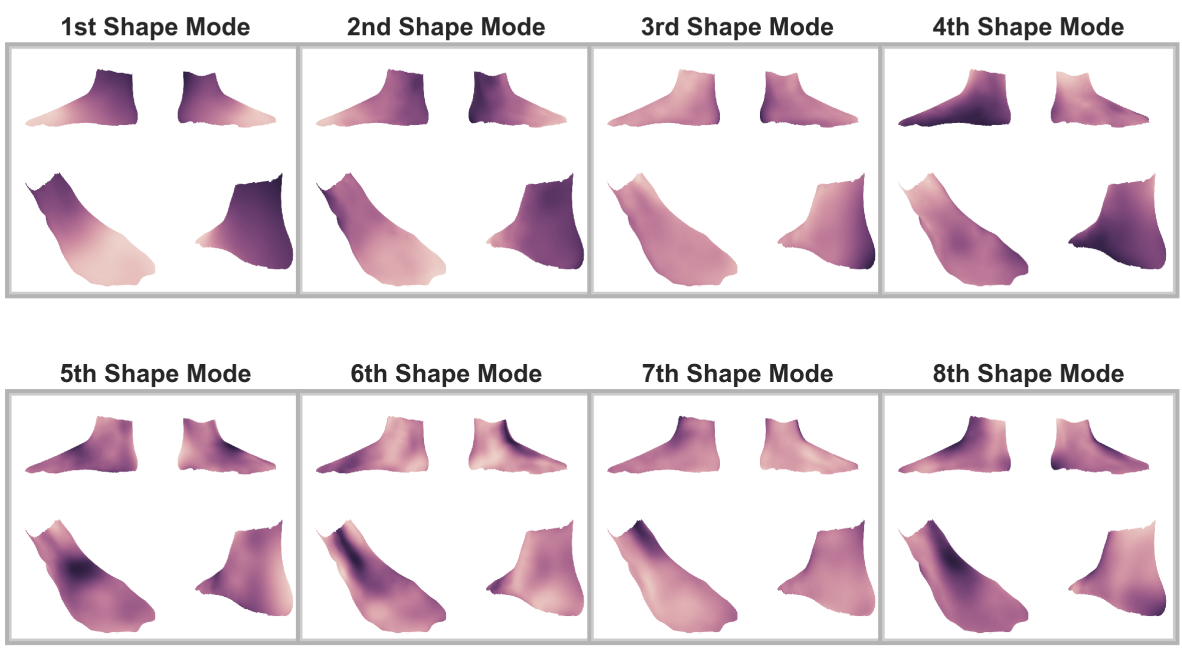

Shape mode normalized correlation

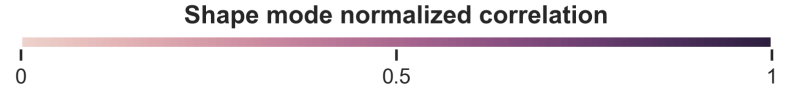

Figure 6: Each shape mode's principal axis represented as a heatmap overlaid on the mean foot and shown from 4 different point-of-views. The darker regions represent vertices which are most correlated with the shape mode's principal axis, and therefore see deformations in the shape mode. 


\section{Deformations along each shape mode's axis}
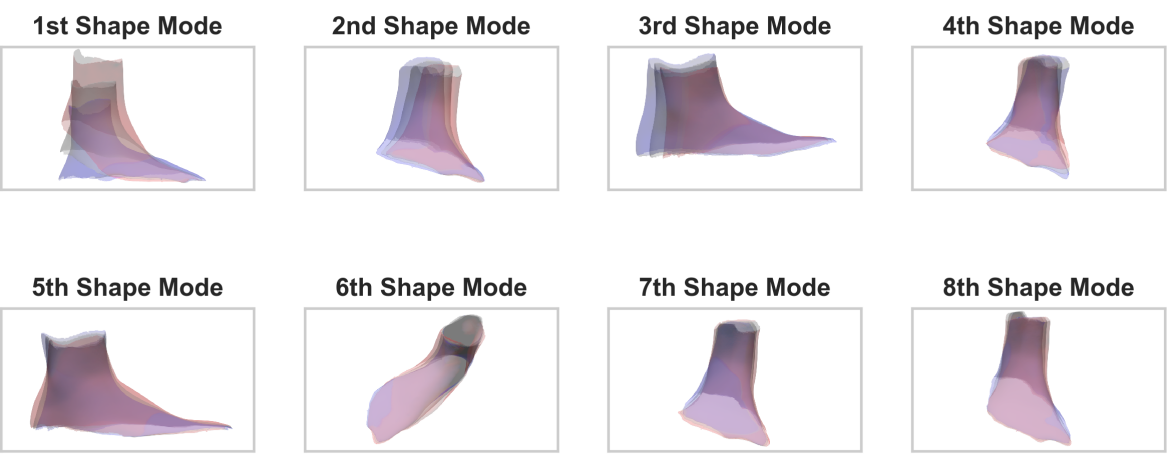

Shape Mode Representations

-2 std. dev. mean foot +2 std. dev.

Figure 7: Foot shape deformation at +2 and -2 standard deviations along each shape mode's principal axis, overlaid on the mean foot. The point-of-view is set to highlight the major variance along each shape mode's axis. 


\section{Acknowledgements}

The authors would like to thank Rodger Kram for providing the laboratory space and treadmill used in the study, Wouter Hoogkamer for assistance with equipment setup, and Steven Priddy for assistance isolating the right foot from the $4 \mathrm{D}$ scans. The authors would also like to thank Brian Corner and Matthew Reed for providing a highquality averaged foot-scan to be used as the template for registration. This project was supported with a National Science Foundation Graduate Research Fellowship Grant DGE 1650115.

\section{References}

Allen, B., Curless, B., Popović, Z., 2003. The space of human body shapes: Reconstruction and parameterization from range scans. ACM Transactions on Graphics 22, 587-594. doi 10.1145/882262.882311.

Anguelov, D., Srinivasan, P., Koller, D., Thrun, S., Rodgers, J., Davis, J., 2005. SCAPE: Shape Completion and Animation of People. ACM Transactions on Graphics 24, 408-416. doi 10.1145/1073204.1073207.

ASTM F539-01, 2017. Standard Practice for Fitting Athletic Footwear. Technical Report. ASTM. West Conshohocken, PA. doi 10.1520/F0539-01R17.

Barisch-Fritz, B., Schmeltzpfenning, T., Plank, C., Grau, S., 2014a. Foot deformation during walking: Differences between static and dynamic 3D foot morphology in developing feet. Ergonomics 57, 921-933. URL: http:// www.tandfonline.com/doi/abs/10.1080/00140139.2014.899629, doi:10. 1080/00140139.2014.899629.

Barisch-Fritz, B., Schmeltzpfenning, T., Plank, C., Hein, T., Grau, S., 2014b. The effects of gender, age, and body mass on dynamic foot shape and foot deformation in children and adolescents. Footwear Science 6, 27-39. doi: 10 . 1080/19424280.2013.834982.

Bernardini, F., Mittleman, J., Rushmeier, H., Silva, C., Taubin, G., 1999. The Ball-Pivoting Algorithm for Surface Reconstruction. IEEE Transactions on Visualization and Computer Graphics 5, 349-359.

Boppana, A., Anderson, A.P., 2019. DynaMo: Dynamic Body Shape and Motion Capture with Intel RealSense Cameras. The Journal of Open Source Software 4. URL: https://doi.org/10.21105/joss.01466, doi 10.21105/ joss.01466.

Browning, R.C., Baker, E.A., Herron, J.A., Kram, R., 2006. Effects of obesity and sex on the energetic cost and preferred speed of walking. Journal of Applied Physiology 100, 390-398. doi 10.1152/japplphysiol.00767.2005. 
Chen, Y., Medioni, G., 1992. Object modelling by registration of multiple range images. Image and Vision Computing 10, 27242729. URL: https://graphics.stanford.edu/\{ $\}$ smr/ICP/comparison/ chen-medioni-align-rob91.pdf.

Conrad, B.P., Amos, M., Sintini, I., Polasek, B.R., Laz, P., 2019. Statistical shape modelling describes anatomic variation in the foot. Footwear Science 11, S203-S205. URL: https://www.tandfonline.com/doi/full/10.1080/ 19424280.2019 .1606334 doi $10.1080 / 19424280.2019 .1606334$.

Dobson, J.A., Riddiford-Harland, D.L., Bell, A.F., Steele, J.R., 2018. The threedimensional shapes of underground coal miners' feet do not match the internal dimensions of their work boots. Ergonomics 61, 588-602. URL: http:// doi.org/10.1080/00140139.2017.1397201, doi:10.1080/00140139.2017. 1397201

Fischler, M.A., Bolles, R.C., 1981. Random sample consensus: A Paradigm for Model Fitting with Applications to Image Analysis and Automated Cartography. Communications of the ACM 24, 381-395. doi $10.1145 / 358669.358692$

Gefen, A., Megido-Ravid, M., Itzchak, Y., Arcan, M., 2000. Biomechanical analysis of the three-dimensional foot structure during gait: A basic tool for clinical applications. Journal of Biomechanical Engineering 122, 630-639. doi $10.1115 / 1.1318904$

Giles, E., Vallandigham, P.H., 1991. Height Estimation from Foot and Shoeprint Length. Journal of Forensic Sciences 36, 13129J. doi:10.1520/jfs13129j.

Gordon, C.C., Blackwell, C.L., Bradtmiller, B., Parham, J.L., Barrientos, P., Paquette, S.P., Corner, B.D., Carson, J.M., Venezia, J.C., Rockwell, B.M., Mucher, M., Kristensen, S., 2014. 2012 Anthropometric Survey of U.S. Army Personnel: Methods and Summary Statistics. Technical Report. ARMY NATICK SOLDIER RESEARCH DEVELOPMENT AND ENGINEERING CENTER MA. Natick, MA. URL: https://apps.dtic.mil/docs/citations/ ADA611869

Grant, T.M., Diamond, L.E., Pizzolato, C., Killen, B.A., Devaprakash, D., Kelly, L., Maharaj, J.N., Saxby, D.J., 2020. Development and validation of statistical shape models of the primary functional bone segments of the foot. PeerJ 2020, e8397. doi $10.7717 /$ peerj.8397.

Grau, S., Barisch-Fritz, B., 2018. Improvement of safety shoe fit - evaluation of dynamic foot structure. Footwear Science 10, 179-187. URL: https:// doi.org/10.1080/19424280.2018.1529062, doi 10.1080/19424280.2018. 1529062 .

Hicks, J., 1954. The mechanics of the foot II. The plantar aponeurosis and the arch. Journal of Anatomy 88, 25-30. 
Jurca, A., Dzeroski, S., 2013. Length dispersion of shoes labelled with the same size in the UK shoe-size system. Footwear Science 5, 2-5. doi 10.1080/ 19424280.2013 .799543 .

Jurca, A., Žabkar, J., Džeroski, S., 2019. Analysis of 1.2 million foot scans from North America, Europe and Asia. Scientific Reports 9, 1-10. doi10.1038/ s41598-019-55432-z.

Kessler, S.E., Lichtwark, G.A., Welte, L.K., Rainbow, M.J., Kelly, L.A., 2020. Regulation of foot and ankle quasi-stiffness during human hopping across a range of frequencies. Journal of Biomechanics , 109853URL: https: //doi.org/10.1016/j.jbiomech.2020.109853, doi:10.1016/j.jbiomech. 2020.109853 .

Kim, K.H., Young, K.S., Bernal, Y., Boppana, A., Vu, L.Q., Benson, E.A., Jarvis, S., Rajulu, S.L., 2016. A Parametric Model of Shoulder Articulation for Virtual Assessment of Space Suit Fit, in: Proceedings of the 7th International Conference on 3D Body Scanning Technologies, Lugano, Switzerland. pp. 201-207. URL: https://ntrs.nasa.gov/search.jsp?R=20160012730, doi:10.15221/16.201.

Kouchi, M., Kimura, M., Mochimaru, M., 2009. Deformation of foot cross-section shapes during walking. Gait and Posture 30, 482-486. URL: http://www.sciencedirect.com/science/article/pii/ S0966636209003087, doi $10.1016 / j$.gaitpost.2009.07.113

Krauss, I., Grau, S., Mauch, M., Maiwald, C., Horstmann, T., 2008. Sex-related differences in foot shape. Ergonomics 51, 1693-1709. URL: https://doi. org/10.1080/00140130802376026 doi $10.1080 / 00140130802376026$.

Krauss, I., Valiant, G., Horstmann, T., Grau, S., 2010. Comparison of female foot morphology and last design in athletic footwear-are men's lasts appropriate for women? Research in Sports Medicine 18, 140-156. doi10.1080/ 15438621003627216 .

Leardini, A., Benedetti, M.G., Berti, L., Bettinelli, D., Nativo, R., Giannini, S., 2007. Rear-foot, mid-foot and fore-foot motion during the stance phase of gait. Gait and Posture 25, 453-462. URL: http://www. sciencedirect.com/science/article/pii/S0966636206001603, doi 10 . $1016 / j$.gaitpost.2006.05.017

Lundgren, P., Nester, C., Liu, A., Arndt, A., Jones, R., Stacoff, A., Wolf, P., Lundberg, A., 2008. Invasive in vivo measurement of rear-, mid- and forefoot motion during walking. Gait and Posture 28, 93-100. doi 10.1016/ j.gaitpost.2007.10.009.

Luo, G., Stergiou, P., Worobets, J., Nigg, B., Stefanyshyn, D., 2009. Improved footwear comfort reduces oxygen consumption during running. Footwear Science $1,25-29$. doi $10.1080 / 19424280902993001$ 
Luximon, Y., Luximon, A., 2013. Sizing and grading of shoe lasts. Handbook of Footwear Design and Manufacture , 197-215doi:10.1533/9780857098795. 3.197 .

Martínez-Martínez, J.M., Martín-Guerrero, J.D., Soria-Olivas, E., Bernabeu, J.A., Escandell-Montero, P., Stark, R.H., Serrano-López, A.J., Montiel, E., 2017. Use of SOMs for footwear comfort evaluation. Neural Computing and Applications 28, 1763-1773. URL: https://doi.org/10.1007/ s00521-015-2139-x, doi $10.1007 / \mathrm{s} 00521-015-2139-\mathrm{x}$.

Mündermann, A., Stefanyshyn, D.J., Nigg, B.M., 2001. Relationship between footwear comfort of shoe inserts and anthropometric and sensory factors. Medicine and Science in Sports and Exercise 33, 1939-1945. doi 10.1097/ 00005768-200111000-00021.

Oladipo, G., Bob-Manuel, I., Ezenatein, G., 2008. Different Weight Bearing Conditions Amongst Nigerians. The Internet Journal of Biological Anthropology $3,1-7$.

Park, B.K., Reed, M.P., 2015. Parametric body shape model of standing children aged 311 years. Ergonomics 58, 1714-1725. URL: https://doi.org/10. 1080/00140139.2015.1033480, doi 10.1080/00140139.2015.1033480

Park, B.K.D., Ebert, S., Reed, M.P., 2017. A parametric model of child body shape in seated postures. Traffic Injury Prevention 18, 533-536. URL: https://doi.org/10.1080/15389588.2016.1269173, doi10.1080/ 15389588.2016.1269173.

Pedregosa, F., Weiss, R., Brucher, M., Varoquaux, G., Gramfort, A., Michel, V., Thirion, B., Grisel, O., Blondel, M., Prettenhofer, P., Weiss, R., Dubourg, V., Vanderplas, J., Passos, A., Cournapeau, D., Brucher, M., Perrot, M., Duchesnay, É., Buitinck, L., Louppe, G., Grisel, O., Pedregosa, F., Mueller, A., 2011. Scikit-learn: Machine Learning in Python. Journal of Machine Learning Research 12, 2825-2830. URL: http://jmlr.csail.mit.edu/papers/ v12/pedregosa11a.html\{\%\}5Cnhttp://arxiv.org/abs/1201.0490, doi:10.1145/2786984.2786995, arXiv:1201.0490.

Price, C., Nester, C., 2016. Foot dimensions and morphology in healthy weight, overweight and obese males. Clinical Biomechanics 37, 125-130. doi 10.1016/ j.clinbiomech.2016.07.003.

Reed, M., Raschke, U., Tirumali, R., Parkinson, M., 2014. Developing and Implementing Parametric Human Body Shape Models in Ergonomics Software. 3rd Digital Human Modeling Symposium , 1-8.

Reed, M.P., Ebert, S.M., Corner, B.D., 2013. Statistical Analysis to Develop a Three-Dimensional Surface Model of a Midsize-Male Foot. Technical Report October. US Army TARDEC. 
Reed, M.P., Parkinson, M.B., 2008. Modeling variability in torso shape for chair and seat design. Proceedings of the ASME Design Engineering Technical Conference 1, 561-569. doi 10.1115/DETC2008-49483

Riebe, D., Franklin, B.A., Thompson, P.D., Garber, C.E., Whitfield, G.P., Magal, M., Pescatello, L.S., 2015. Updating ACSM's recommendations for exercise preparticipation health screening. Medicine and Science in Sports and Exercise 47, 2473-2479. URL: https://insights.ovid.com/crossref?an= 00005768-201511000-00028, doi 10.1249/MSS.0000000000000664.

Rusu, R.B., Cousins, S., 2011. 3D is here: Point Cloud Library (PCL), in: Proceedings - IEEE International Conference on Robotics and Automation, Shanghai, China. URL: http://pointclouds .org, doi:10.1109/ICRA.2011. 5980567.

Scott, S.H., Winter, D.A., 1993. Biomechanical model of the human foot: Kinematics and kinetics during the stance phase of walking. Journal of Biomechanics 26, 1091-1104. doi 10.1016/S0021-9290(05)80008-9.

Stanković, K., Huysmans, T., Danckaers, F., Sijbers, J., Booth, B.G., 2020. Subject-specific identification of three dimensional foot shape deviations using statistical shape analysis. Expert Systems with Applications 151, 113372. doi:10.1016/j.eswa.2020.113372.

Tomassoni, D., Traini, E., Amenta, F., 2014. Gender and age related differences in foot morphology. Maturitas 79, 421-427. URL: http://dx.doi.org/10. $1016 /$ j.maturitas.2014.07.019 doi $10.1016 / j$.maturitas.2014.07.019

Van den Herrewegen, I., Cuppens, K., Broeckx, M., Barisch-Fritz, B., Vander Sloten, J., Leardini, A., Peeraer, L., 2014. Dynamic 3D scanning as a markerless method to calculate multi-segment foot kinematics during stance phase: Methodology and first application. Journal of Biomechanics 47, 2531-2539. URL: http://www.sciencedirect.com/science/article/pii/ S0021929014003492, doi $10.1016 / \mathrm{j} \cdot$ jbiomech.2014.06.010

Wannop, J.W., Stefanyshyn, D.J., Anderson, R.B., Coughlin, M.J., Kent, R., 2019. Development of a Footwear Sizing System in the National Football League. Sports Health 11, 40-46. doi 10.1177/1941738118789402.

Wolf, P., Stacoff, A., Liu, A., Nester, C., Arndt, A., Lundberg, A., Stuessi, E., 2008. Functional units of the human foot. Gait and Posture 28, 434-441. doi $10.1016 /$ j.gaitpost.2008.02.004

Wunderlich, R.E., Cavanagh, P.R., 2001. Gender differences in adult foot shape: Implications for shoe design. Medicine and Science in Sports and Exercise 33, 605-611. URL: https://insights.ovid.com/crossref?an= 00005768-200104000-00015, doi:10.1097/00005768-200104000-00015. 
Xiong, S., Goonetilleke, R.S., Zhao, J., Li, W., Witana, C.P., 2009. Foot deformations under different load-bearing conditions and their relationships to stature and body weight. Anthropological Science 117, 77-88. doi 10.1537/ ase.070915.

Yeo, I., Johnson, R.A., 2000. A new family of power transformations to improve normality or symmetry. Biometrika 87, 954-959. URL: https://doi.org/ 10.1093/biomet/87.4.954, doi $10.1093 /$ biomet/87.4.954

Zelik, K.E., Honert, E.C., 2018. Ankle and foot power in gait analysis: Implications for science, technology and clinical assessment. Journal of Biomechanics 75, 1-12. URL: https://doi.org/10.1016/j.jbiomech.2018.04.017, doi:10.1016/j.jbiomech.2018.04.017.

Zhang, J., Hislop-Jambrich, J., Besier, T.F., 2016. Predictive statistical models of baseline variations in 3-D femoral cortex morphology. Medical Engineering and Physics 38, 450-457. URL: http://dx.doi.org/10.1016/j.medengphy. 2016.02.003, doi $10.1016 / j$.medengphy.2016.02.003

Zhou, Q.Y., Park, J., Koltun, V., 2018. Open3D: A Modern Library for 3D Data Processing. arXiv:1801.09847 URL: http://arxiv.org/abs/1801.09847, arXiv:1801.09847.

Zou, H., Hastie, T., 2005. Regularization and variable selection via the elastic net. Journal of the Royal Statistical Society. Series B: Statistical Methodology 67, 301-320. doi $10.1111 / \mathrm{j} .1467-9868.2005 .00503 . \mathrm{x}$ 
Supplemental Information

\section{Appendix I : Supplemental Methods}

3 Following is more details on the mesh construction, template registration, 4 and joint angle calculation methods.

5 0.1. Mesh Construction

The $\mathrm{C}++$ implementation of the PointCloud Library (Rusu and Cousins, 2011) was used to identify and isolate the right foot from the point set. First, the point clouds were downsampled with a voxel size of $3 \mathrm{~mm}$ to reduce required 9 computing power. A RANSAC algorithm (Fischler and Bolles, 1981) was use to to identify the flat treadmill floor with a plane model, and remove it from the 1 point cloud. Euclidean cluster extraction was then used to detect the poin ${ }_{12}$ clusters that make up each foot. The total color value of each point cluster was
${ }_{13}$ used to identify the right foot from the left foot, as the left foot had a lower 13 used to identify the right foot from the left foot, as the left foot had a lower
${ }_{14}$ total color value due to the black sock. The left foot was then removed from 15 the point cloud, leaving only the right foot for processing.

${ }_{16}$ Poisson surface reconstruction was done using Open3D (Zhou et al., 2018) 7 this adds a topological layer interpreted from the pointcloud. Point norma ${ }_{18}$ were calculated for the point cloud using the 10 nearest neighbors. A ball9. pivoting algorithm (Bernardini et al., 1999) is then used with the point norma 20 to estimate the surface from the point cloud and construct the foot scan mesh.

21 0.2. Foot Template Registration

From the provided template, the toes were smoothed into a single structure and parts of the upper shank removed to be better fit to the captured data, with 24 a finalized structure of 29873 points. The overall registration process follows a 25 three-step process: a rough alignment followed by two radial-basis function ${ }_{26}$ (RBF) fine alignment steps

The registration process was first completed for each subject's data with a ${ }_{28}$ foot scan mesh manually identified near mid-stance. A point-to-plane iterative29 closest-point (ICP) algorithm (Chen and Medioni, 1992) was used to roughly 30 align the template foot to the scan mesh with the Open3D library (Zhou et al., 31 2018).

Corresponding points between both the scan mesh and the ICP-aligned tem33 plate were found using a radial-search KD-Tree implemented in the Open3D ${ }_{34}$ library (Zhou et al., 2018). Any points on the scan mesh which were not within $351 \mathrm{~cm}$ of a corresponding point on the aligned template were deleted; these points 
36 represented parts of the treadmill floor which were missed in the RANSAC iden37 tification and parts of the upper shank. Similarly, any points on the template 38 not within $1 \mathrm{~cm}$ of a corresponding point on the scan mesh were temporarily set 39 aside from the template; these points correspond to those near holes in the scan 40 mesh which would be refilled in later processing

Thin-plate spline RBFs have been used to surface fit templates to scanned a2 body shapes (Park and Reed, 2015), and so were used in two stages in this re43 search. A first-pass RBF registration, using a thin-plate spline for interpolation, 4 was done between the template and the scan using the GIAS2 package (Zhan s. et al., 2016) To prevent overfitting of the RBF to the noise on the edges of the captured pointeloul, a maxim of ${ }^{6}$ captured pointcloud, a maximum or five iterations were done on the first-pas RBF rel poith then chpenderme 9 diate template so unknown sections (e.g. holes in the scan data), taking the value of the template. 51 However, the disparity between the known and unknown sections created majo 52 discrepencies in the morphed template not representative of the scan data. A second-pass RBF registration was done from the ICP-aligned template ${ }_{54}$ to the intermediate template with the same parameters as the first-pass regiss5 tration. This smooths out the unknown sections representing holes in the scan 6 data with the surrounding known sections. The second-pass registered template 57 was saved as the final registered template.

Following the registration of the mid-stance scan, the process was repeated 59 both forwards towards toe-off and backwards toward heel-strike on a scan-byso scan basis. In this iterative fashion, the previous scan's registered template was 61 used as the template for the following scan. During the iterative registration 62 process, the RBF alignment was only conducted for one iteration for both the ${ }_{62}$ process, the RBF alignment was only conducted

64 0.3. Joint Angle Calculation

The original template identified the lateral malleolus, medial malleolus, $1 \mathrm{~s}$ 66 metatarsal head, 5 th metatarsal head, and 2 nd toe landmarks as certain vertices. 67 New landmark vertices for the lateral shank and medial shank were manually 68 picked on the template.

Post-registration scans were aligned to a common coordinate frame based around the toes. The origin was defined as the point along the vector from the 1st metatarsal head landmark to the 5 th metatarsal head landmark which orthogonal to the second phalange. From the origin the $x$-axis, was wef 2 3 as pointing towards the 2 nd toe. The y-axis, was pointed towards the 5 th 4 metatarsal. The z-axis was the cross-product of both $x$ - and y-axes, pointed 6 the MTP join

The ankle joint center was defined as the midpoint between the medial and s lateral malleous. The ankle's local z-axis is aligned vertically with the shan 7 center, defined as the center between the lateral shank and medial shank landso marks. The ankle's local y-axis is aligned from the shank center to the lateral 
1 malleolus. The ankle's $x$-axis is the cross-product of the $y$-and z-axis, pointed in the forward direction towards the toes.

Static reference angles were taken from these coordinate systems at mid-

4 stance. For the ankle joint, the z-axis served as the internal/external rotation ${ }_{35}$ axis, the $y$-axis as the dorsi/plantarflexion axis, and the $\mathrm{x}$-axis as the invers6 sion/eversion axis. Since the model's origin was at the toes, the calculation 7 for MTP dorsi/plantarflexion was modified. The new local MTP joint coordis nate system had the $\mathrm{x}$-axis defined as pointing from the ankle joint center to 9 the MTP joint center, as such the y-axis represented MTP dorsi/plantarflexion. Since there is little flexibility in the transverse and frontal planes of the MTP joint, the xaxis thereforestest whole foot inversion/eversion, and the joint, the $\mathrm{x}$-axis therefore represented whole foot inversion/eversion, and the $\mathrm{z}$ ${ }_{2}$ axis represented whole foot internal/external rotation around the origin. MTP (ne Euler angle 4 difference from the static joint coordinate system around each axis. Each sub${ }^{45}$ ject's joint angles are low-pass filtered with a 2nd order low-pass Butterworth 96 filter with a cutoff frequency of $15 \mathrm{~Hz}$. The global and local coordinate systems 97 are summarized in Fig. 1.

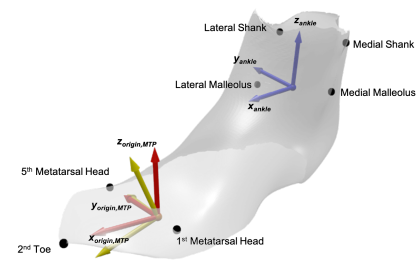

Figure 1: Coordinate system defined from registered scans. Anatomical landmarks are shown The ankle joint's local coordinate system is shown in blue, the MTP joint's in red. Directions for each coordinate system are shown in bold text

\section{Reference}

99 Bernardini, F., Mittleman, J., Rushmeier, H., Silva, C., Taubin, G., 1999. The ${ }_{100}$ Ball-Pivoting Algorithm for Surface Reconstruction. IEEE Transactions on ${ }_{101}$ Visualization and Computer Graphics 5, 349-359.

102 Chen, Y., Medioni, G., 1992. Object modelling by registration of ${ }_{103}$ multiple range images. Image and Vision Computing 10, 2724 
104 2729. URL: https://graphics.stanford.edu/ $\{\sim\} \mathrm{smr} / \mathrm{ICP} /$ comparison/ chen-medioni-align-rob91.pdf

${ }_{106}$ Fischler, M.A., Bolles, R.C., 1981. Random sample consensus: A Paradigm for

${ }_{107}$ Model Fitting with Applications to Image Analysis and Automated Cartogra${ }_{108}$ phy. Communications of the ACM 24, 381-395. doi:10.1145/358669.358692.

109 Park, B.K., Reed, M.P., 2015. Parametric body shape model of standing children aged 3-11 years. Ergonomics 58, 1714-1725. URL: https://doi.org/10.
1080/00140139.2015.1033480, doi:10.1080/00140139.2015.1033480.

112 Rusu, R.B., Cousins, S., 2011. 3D is here: Point Cloud Library (PCL), in: Proceeding - IEEE Intertion Shanghai, China. URL: http://pointclouds org, doi:10.1109/ICRA.2011. 5980567 .

16 Zhang, J., Hislop-Jambrich, J., Besier, T.F., 2016. Predictive statistical models of baseline variations in 3-D femoral cortex morphology. Medical Engineering and Physics 38, 450-457. URL: http://dx. doi .org/10.1016/j .medengphy . 192016.02 .003 , doi:10.1016/j.medengphy . 2016.02.003

120 Zhou, Q.Y., Park, J., Koltun, V., 2018. Open3D: A Modern Library for 3D Data

${ }^{121}$ Processing. arXiv:1801.09847 URL: http://arxiv.org/abs/1801.09847,

aryiv: 1801.09847. 
L. 


\begin{abstract}
${ }^{124}$ Appendix III : Supplemental Video
125 https://youtu.be/XshzgabhmNE

126 The attached video shows the predictive capability of the developed para-

${ }_{227}$ metric statistical shape model. The sliders predicts principal component scores,

28 which are then inverse-transformed into a foot shape. The stance phase slider

${ }_{29}$ predicts joint angles during the stance phase to visualize foot morphology changes

${ }_{130}$ during stance phase.
\end{abstract}

